\title{
Neuroethologic differences in sleep deprivation induced by the single- and multiple-platform methods
}

R. Medeiros ${ }^{1}$,

C. Lenneberg-Hoshino², K. Hoshino ${ }^{2}$ and S. Tufik ${ }^{1}$
'Departamento de Psicobiologia, Universidade Federal de São Paulo, São Paulo, SP, Brasil

${ }^{2}$ Departamento de Ciências Biológicas, Faculdade de Ciências, Universidade Estadual Paulista, Bauru, SP, Brasil
Correspondence

\section{S. Tufik}

Departamento de Psicobiologia EPM, UNIFESP

Rua Botucatu, 862

04023-062 São Paulo, SP

Brasil

Fax: 55 (011) 572-5092

Research supported by Associação Fundo e Incentivo à Psicofarmacologia (AFIP). R. Medeiros and C. Lennenberg-Hoshino are the recipients of AFIP and CAPES fellowships, respectively. Publication supported by FAPESP.

\section{Abstract}

It has been proposed that the multiple-platform method (MP) for desynchronized sleep (DS) deprivation eliminates the stress induced by social isolation and by the restriction of locomotion in the singleplatform (SP) method. MP, however, induces a higher increase in plasma corticosterone and ACTH levels than SP. Since deprivation is of heuristic value to identify the functional role of this state of sleep, the objective of the present study was to determine the behavioral differences exhibited by rats during sleep deprivation induced by these two methods. All behavioral patterns exhibited by a group of 7 albino male Wistar rats submitted to 4 days of sleep deprivation by the MP method (15 platforms, spaced $150 \mathrm{~mm}$ apart) and by 7 other rats submitted to sleep deprivation by the SP method were recorded in order to elaborate an ethogram. The behavioral patterns were quantitated in 10 replications by naive observers using other groups of 7 rats each submitted to the same deprivation schedule. Each quantification session lasted $35 \mathrm{~min}$ and the behavioral patterns presented by each rat over a period of $5 \mathrm{~min}$ were counted. The results obtained were: a) rats submitted to the MP method changed platforms at a mean rate of 2.62 \pm 1.17 platforms $\mathrm{h}^{-1}$ animal $^{-1}$; b) the number of episodes of noninteractive waking patterns for the MP animals was significantly higher than that for SP animals (1077 vs 768); c) additional episodes of waking patterns ( $26.9 \pm 18.9$ episodes/session) were promoted by social interaction in MP animals; d) the cumulative number of sleep episodes observed in the MP test (311) was significantly lower (chisquare test, 1 d.f., $\mathrm{P}<0.05$ ) than that observed in the SP test (534); e) rats submitted to the MP test did not show the well-known increase in ambulatory activity observed after the end of the SP test; f) comparison of 6 MP and 6 SP rats showed a significantly shorter latency to the onset of DS in MP rats $(7.8 \pm 4.3$ and $29.0 \pm 25.0 \mathrm{~min}$, respectively; Student t-test, $\mathrm{P}<0.05$ ). We conclude that the social interaction occurring in the MP test generates additional stress since it increases the time of forced wakefulness and reduces the time of rest promoted by synchronized sleep.
Key words

- Sleep deprivation methods

- Stress

- Social interactions

- Group crowding effects 
Total or selective sleep deprivation is of heuristic value for the investigation of the functional role of sleep states, which is still unclear despite the large number of studies devoted to the subject. The sleep deprivation method plays a key role in these investigations since valid conclusions can be drawn only in cases where the effects observed can be attributed exclusively to sleep suppression.

Desynchronized sleep (DS), the sleep state during which oniric activity occurs in man and other mammals (1), has been suppressed in rats by the classical single-platform method (SP) (2), since the animal kept on the platform falls into the surrounding water when the muscular atonia of DS sets in. Hyperthermia (3), hypothermia (4), decreased memory consolidation (5), increased aggressiveness (6), suppression of immunological responses (7) and many other effects have been reported to result from such deprivation. On the basis of these effects, some important hypotheses have been raised concerning the role played by DS, such as memory consolidation (5) or thermoregulatory (8) functions. The question, however, remains unanswered since the stress involved in the deprivation method may be the causative factor of these deprivation effects. In fact, the stress of social isolation in animals with previous social experience increases the circulating levels of corticosterone (9), promotes immunosuppression (10), and increases aggressive behavior (11), as observed after DS deprivation. Space limitation which reduces the locomotion area also increases plasma corticosterone levels (12).

The multiple-platform method (MP), which involves many disposable platforms and suppresses DS simultaneously in a group of rats, has been used to avoid the stresses of social isolation and locomotion restriction (12). However, plasma adrenocorticotrophic hormone (ACTH) and corticosterone levels and adrenal weights of rats submitted to sleep deprivation by the MP method were higher $(13,14)$ than those observed in rats submitted to the SP test, a fact confirmed more recently by other members of our group (Sucheki D and Tufik S, unpublished results) using large platform controls and daily hormone evaluations. These data indicate that, unexpectedly, the MP method involves more stress than the SP method. This assumption seems valid since the effects of sleep deprivation, such as fighting behavior, are significantly increased in the MP method (14).

In view of the heuristic importance of methodological problems in terms of the strategy used to identify the functional role of DS, the objective of the present study was to determine the factor(s) responsible for the increased signs of stress observed in the MP method. Behavioral aspects observed during DS deprivation in MP and SP animals were compared since the mechanical process used to suppress sleep is the same for both methods. A large platform was not used as a control because recent studies show that it deprived DS equally as smaller stands (15).

Seven albino male Wistar rats weighing 250-350 g, 3-4 months of age, were submitted to 4-day DS deprivation by the MP method, and 7 other similar animals were submitted to 4-day DS deprivation by the SP method. During deprivation, the animals were videotaped in order to record their behavioral patterns and to prepare a protocol for their quantification. The rats were identified by a number painted on their backs with hair dye. The MP device used for DS deprivation consisted of 15 cylindrical $60-\mathrm{mm}$ high platforms measuring $50 \mathrm{~mm}$ in diameter, spaced $150 \mathrm{~mm}$ apart and fixed in a plastic cage measuring $1050 \times 550 \times 270 \mathrm{~mm}$. The cage was filled with water up to $10 \mathrm{~mm}$ from the platform tops. The SP device for DS deprivation was an individual $400 \times 340 \times 160-$ $\mathrm{mm}$ plastic cage containing only one platform surrounded by water up to the same level as in the MP device. All observations were made in a room with controlled tem- 
perature $\left(24-25^{\circ} \mathrm{C}\right)$ and light (7:00-19:00 h). Commercial pellets were available ad libitum in a feeder inside the cage lid and water was supplied through conventional drinking spouts and renewed daily between 8:00 and 9:00 a.m.

The behavioral patterns detected during deprivation were assigned to the following categories: a) awake noninteractive patterns consisting of grooming, orienting reflex, quiet immobility, exploratory locomotion, rearing, sniffing, spontaneous walking inside water, feeding, cage cover gnawing, and switching platforms; b) interactive awake patterns consisting of unilateral or reciprocal approximation, sniffing, touching, social grooming, standing, climbing conspecifics, displacement, flight, escape, repelling, fighting, persecuting and wetting conspecifics; c) noninteractive sleep episodes characterized by relative immobility, eyes closed, resting postures and indifference to habitual stimuli; d) interactive sleep episodes defined as sleeping behavior, climbing or being climbed on by another animal using the same platform or being held up by another rat occupying a neighboring platform; e) sleep-related patterns consisting of a small postural change or correction without eye opening, and a sequence named NTW consisting of a brief awakening after a slow progressive head dropping, brief touching of the water surface with the nose and quick posture correction. The brief awakenings of these sleep-related behaviors were not considered to interrupt sleep, except when other behavioral patterns prolonged wakefulness for more than $2 \mathrm{~s}$.

The frequencies of the behavioral patterns along 4 days of DS deprivation were quantified by two naive observers using a new batch of 14 rats kept on the same deprivation schedule as used before. Behavioral watching sessions lasted $35 \mathrm{~min}$ each and three daily sessions were held (at 11:0012:10, 14:00-15:10 and 17:00-18:10 h), except for the last day when only one observation was made (a total of 10 observation sessions). Observations were restricted to the light phase of the day which corresponds to the main resting period of rats. Since evaluation of the behaviors exhibited by all rats in the group at the same time was extremely difficult, the patterns exhibited by each animal over a period of $5 \mathrm{~min}$ were counted. The mean frequency was used in cases of discrepant counts made by the observers. Cumulative numbers of behavioral episodes for the group per observation session or total numbers of episodes along the deprivation period were computed for analysis.

Since observations made after the end of deprivation indicated differences in sleep onset latency, a group of 12 rats was chronically implanted with electrodes for electrocorticographic and electromyographic recordings using previously reported procedures $(1,2)$. After postoperative recovery, the animals were submitted to MP $(\mathrm{N}=6)$ or to SP $(\mathrm{N}=6)$ deprivation for 4 days and electrographic recordings were started immediately after the end of deprivation in order to determine the latencies to the onset of sleep states.

The total frequencies of each behavioral category counted during sleep deprivation are presented in Table 1. The total number of

Table 1 - Total frequencies of waking and sleep behavioral patterns exhibited by rats during desynchronized sleep deprivation by the single- and multiple-platform methods.

The frequencies were obtained by summing all pattern counts made in 10 observation sessions of 35 min each along 4 days of deprivation. The behavioral patterns exhibited by each rat were counted over a period of $5 \mathrm{~min}$ and the total for the group $(N=7)$ in the session was computed. The behavioral patterns that compose each category are described in the Methods section. Interactive sleep episodes were included in the total number of sleep episodes for statistical comparison. ${ }^{*} \mathrm{P}<0.05$ compared to single platform (chi-square test, 1 d.f.).

\begin{tabular}{lcc}
\hline & Single platform & Multiple platform \\
\hline Noninteractive waking patterns & 768 & $1077^{*}$ \\
Noninteractive sleep episodes & 534 & $311^{*}$ \\
Interactive waking patterns & - & 269 \\
Interactive sleep episodes & - & 12 \\
Sleep-related patterns & 571 & $256^{*}$
\end{tabular}


Figure 1 - Frequencies of sleep episodes in the single- (SP) and multiple- (MP) platform methods used for desynchronized sleep deprivation in rats. Data are reported as total number of episodes of the group per observation session. Three observation sessions were held per day, and a 10th one was held on the fourth day of deprivation. The number of episodes corresponds to the cumulative counts for 7 rats per group for 5 min periods for each animal.

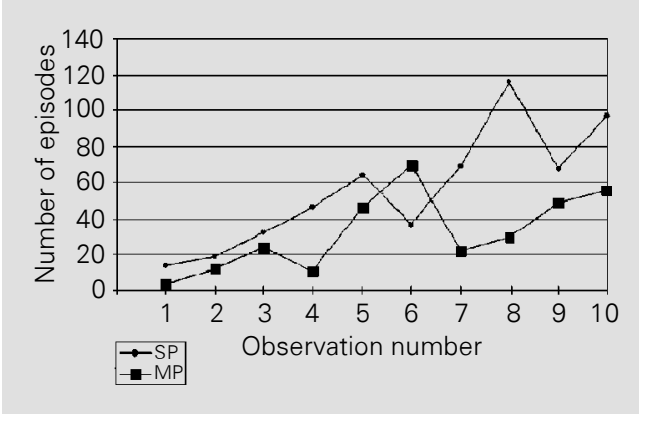

sleep episodes in the MP method was lower than in the SP method (chi-square test, 1 d.f., $\mathrm{P}<0.05$ ). The mean number of sleep episodes per group per observation session in the MP method ( $32.3 \pm 21.5$ per observation) was also lower compared to that observed in the SP method (53.4 \pm 30.3$)$. Figure 1 illustrates inter-observer differences in the number of sleep episodes along deprivation. NTW frequency was significantly lower in MP animals (8.2 \pm 7.1 episodes/observation) than in $\mathrm{SP}$ rats $(38.3 \pm 30.8)$ (Student t-test, $\mathrm{P}<$ $0.05)$. This pattern, which indicates the duration of the sleep episode, started to be manifested after the end of the first day of deprivation by SP animals, whereas it appeared only after the second day in MP animals. Rats submitted to the MP method generally stayed together on neighboring platforms but one or two animals occasionally stayed alone on distant platforms, where they remained undisturbed for long periods of time. The sleep of a rat climbing another animal was interrupted frequently by loss of balance, gross mutual posture corrections or avoidance behaviors and these interruptions shortened the sleep episodes.

Socially induced awakenings frequently lasted longer than the physical interaction period due to subsequent interactive and noninteractive behaviors elicited. Socially related waking episodes, possible only in the MP test, reached a mean number of $26.9 \pm$ 18.9 episodes/observation. Conspecific approximation and touching $(0.83 \pm 0.53$ episodes $\mathrm{h}^{-1}$ animal ${ }^{-1}$ ), holding up and attempt- ing to climb another rat during sleep $(1.88 \pm$ 0.85 episodes $\mathrm{h}^{-1}$ animal $^{-1}$ ) and wetting promoted by a conspecific $(0.61 \pm 0.43$ episodes $\mathrm{h}^{-1}$ animal $^{-1}$ ) were common in the MP test. Other less frequent socially induced awakenings were promoted by displacement of platform dwellers, refusal to be climbed, noise or sudden movements made by conspecifics on neighboring platforms, etc. A greater number of falls into water was observed in the MP $(1.78 \pm 2.1$ per observation) than in the SP test $(0.6 \pm 1.3)$ due to attempts to climb conspecifics, disputes for a platform, holding up, etc. Thus, all MP rats were completely wet at the end of the deprivation period. Episodes of platform switching $\left(2.62 \pm 1.17\right.$ switches $^{-1}$ animal $\left.^{-1}\right)$ also contributed to the higher frequency of awake behavioral patterns in the MP test.

The MP animals did not present the conspicuous period of intense locomotor activity before the onset of sleep as observed in SP animals immediately after the end of sleep deprivation. Visual evaluation indicated a latency of 17 min for sleep occurrence in all MP rats and of $31 \mathrm{~min}$ in SP animals. The absence of the increased locomotor activity period preceding the onset of sleep in MP animals was confirmed in the electrographic observations, in which DS sleep latency was $7.8 \pm 4.3 \mathrm{~min}$, a value significantly lower than $29.0 \pm 25.0 \mathrm{~min}$ computed for SP animals ( $\mathrm{P}<0.05$, Student ttest). The first DS episode lasted $6.0 \pm 1.4$ min in MP animals, a significantly longer time than that observed in SP rats $(3.8 \pm 0.9$ min; $P<0.05$, Student t-test).

Considering all of these quantitative differences observed in the DS deprivation methods, it is possible to state that the social interactions of the MP caused a significant increase in the time the animals spent awake. The mean number of 26.9 socially induced awakening episodes/observation corresponds to an approximate total of 46 episodes $/ \mathrm{h}$ or 1106 episodes/day. Since each awakening episode lasted at least $10 \mathrm{~s}$, we may state that 
a minimum of 3 more h/day of forced wakefulness occurred in the MP than in the SP method. This total amount of forced wakefulness seems to exceed the computed value since other episodes that occurred simultaneously in rats that were not under observation were not counted. The higher mean colonic temperature of rats submitted to the MP test (14) supports this additional forced wakefulness time since an increase in body temperature is observed when synchronized sleep deprivation occurs together with DS deprivation (16). The forced awakenings induced by social interactions when the rats become progressively exhausted and have an increased need to sleep can be considered stressful. This additional stress in the MP test prevents any possible reduction of social isolation and locomotor restriction stresses that might be induced by the method, explaining why plasma corticosterone and ACTH levels were higher in animals submitted to the MP test. This conclusion is also supported by recent data indicating that DS rebound compensation is determined by stress (17). The significant increase in the duration of the first DS episode and its shorter latency observed after the end of sleep deprivation in the MP confirm the higher level of stress involved in this method of sleep deprivation.

\section{References}

1. Timo-laria C, Yamashita R, Hoshino K \& Souza-Mello A (1990). Rostrum movements in desynchronized sleep as a prevalent manifestation of dreaming activity in rats. Brazilian Journal of Medical and Biological Research, 23: 617-620.

2. Jouvet-Mounnier D, Vimont-Vicary $P$, Delorme P \& Jouvet M (1964). Étude de la privation selective de la phase paradoxale du sommeil chez le chat. Comptes Rendus de la Societé de Biologie, 158: 756-759.

3. Seabra ML \& Tufik S (1993). Sodium diclofenac inhibits hyperthermia induced by paradoxical sleep deprivation: the possible participation of prostaglandins. Physiology and Behavior, 54: 923-926.
It is well known that a variable may sometimes have quantitatively and/or qualitatively different effects on grouped individuals compared with the same individuals when they are socially isolated. Many animal groups share this property named "group effect". The stressful social interaction in the MP seems to be a group effect similar to the deleterious interactions that increase the lethality of drugs in grouped mice (18), a property used to assess tranquilizing substances (19). In fact, grouping in the MP test reduced survival time in our more recent observations. Locomotor activity inhibition is reported to be an adaptive adjustment to crowding (20) and this kind of group effect seems to be expressed in the absence of hyperactivity observed after the end of deprivation in the MP test. This group effect is probably overcome by the desperate need for a safe and undisturbed place to manifest DS, and this explains why its adaptive expression is not observed during deprivation.

Finally, the conclusion that the MP method of DS deprivation involves an additional stress originating from the social interactions suggests that the answer to the question concerning the functional role played by this state still depends on the solution of methodological problems.
4. Hoshino K (1996). Food deprivation and hypothermia in desynchronized sleep-deprived rats. Brazilian Journal of Medical and Biological Research, 29: 41-46.

5. Fishbein W \& Gutwein BM (1977). Paradoxical sleep and memory storage processes. Behavioral Biology, 19: 425-464.

6. Tufik $S$ (1981). Changes of response to dopaminergic drugs in rats submitted to REM sleep deprivation. Psychopharmacology, 72: 257-260.

7. Kubo C, Sogawa H, Teshima $H$ \& Nakagawa T (1992). The effects of starvation and sleep deprivation on the immunological functions. Japanese Journal of Psychosomatic Medicine, 32: 13-20.

8. Schmidek WR, Zachariassen KE \&
Hammel HT (1983). Total calorimetric measurements during the sleep-wakefulness cycle in the rat. Brain Research, 288: 261-271.

9. Gambardella P, Greco AM, Stichii R, Belotti R \& Di Renzo G (1994). Individual housing modulates daily rhythms of hypothalamic catecholaminergic system and circulating hormones in adult male rats. Chronobiology International, 11: 213-221.

10. Shanks N, Renton C, Zalcman S \& Anismam $\mathrm{H}$ (1994). Influence of change from grouped to individual housing on a Tcell-dependent immune response in mice: antagonism by diazepam. Pharmacology, Biochemistry and Behavior, 47: 497-502.

11. Angulo JA, Ledoux $M$ \& McEween BS 
(1991). Genomic effects of cold and isolation on magnocellular vasopressin mRNAcontaining cells in the hypothalamus of the rat. Journal of Neurochemistry, 56: 2033-2038.

12. Van Hulzen ZJM \& Coenen AML (1981). Paradoxical sleep deprivation and locomotor activity in rats. Physiology and Behavior, 27: 741-744.

13. Suchecki D, Hipólide DC, Lobo LL \& Tufik $S$ (1995). ACTH and corticosterone responses to multiple and single platform techniques-induced REM sleep deprivation. Sleep Research, 24: 455 (Abstract).

14. Nunes Jr G \& Tufik S (1994). Validation of modified multiple platform methods (MPM) of paradoxical sleep deprivation in rats. Sleep Research, 23: 419 (Abstract).

15. Landis CA (1996). Altered sleep patterns with platform method of REM sleep deprivation in rats. Sleep Research, 25: 469 (Abstract).

16. Landis CA, Bergmann BM, Ismail MM \& Rechschaffen A (1992). Sleep deprivation in the rat: XV. Ambient temperature choice in paradoxical sleep deprived rats. Sleep, 15: 13-20.

17. Jouvet M (1994). Paradoxical sleep mechanisms. Sleep, 17: S77-S83.

18. Greenblatt EN \& Osterberg AC (1961).
Correlate of active and lethal effects of exciting drugs in grouped and isolated mice. Journal of Pharmacology and Experimental Therapeutics, 131: 115-119.

19. Salustiano-Filho J, Hoshino K \& Carlini EA (1966). Effects of Cannabis sativa (marihuana) and chlorpromazine on mice as measured by two methods used to evaluate tranquilizing drugs. Medicina et Pharmacologia Experimentalis, 15: 153-162.

20. Edgar DM, Kilduff TS, Martin CE \& Dement WC (1991). Influence of running wheel activity on free-running sleep/wake and drinking circadian rhythm in mice. Physiology and Behavior, 50: 373-378. 